Ann. Biol. anim. Bioch. Biophys., I968, 8 (4), 549-556..

\title{
NOTE PRELIMINAIRE SUR L'ÉVOLUTION DES CONSTITUANTS BIOCHIMIQUES DU FOIE AU COURS DU GAVAGE DE L'OIE (1)
}

avec la collaboration technique de Marie-Rose SAlichon, M. Guezel et Éliane Penot

B. LECLERCQ, G. DURAND, P. DELPECH et J.-C. BLUM

Station de Recherches avicoles et Station centrale de Nutrition, Centre national de Recherches zootechniques, 78 - Jouy-en-Josas Institut national de la Recherche agronomique

\section{SOMMAIRE}

Nous avons étudié l'influence du gavage sur la composition du tissu hépatique de l'oie. La stéatose correspond à un dépôt massif de triglycérides dont les acides gras semblent être essentiellement d'origine endogène (teneur extrêmement faible en acide linoléique). Bien que les quantités de cholestérol libre et estérifié soient respectivement multipliées par 2 et $5^{\circ}$, elles ne représentent qu'une faible part des lipides accumulés dans le foie gras.

Après I 2 jours de gavage, les quantités totales de protéines, d'ARN et de phospholipides contenus dans l'organe ont considérablement augmenté. Il en est de même du rapport $\frac{\mathrm{ARN}}{\mathrm{ADN}}$. Ces faits plaident en faveur d'un accroissement de l'activité de la cellule hépatique. Ni les proportions des divers phospholipides, ni leur composition en acides gras ne subissent de modifications notables ; on peut donc supposer que le métabolisme cellulaire reste coordonné en dépit de l'exacerbation des processus de synthèse.

Les analyses effectuées à la fin de la période de gavage (2I jours) montrent que l'activité du tissu hépatique demeure encore élevée, quoique à cette époque, elle tende quelque peu à diminuer.

\section{INTRODUCTION}

Les stéatoses hépatiques revêtent des aspects extrêmement divers. En I953, un colloque permettait de faire le point sur la Physiologie, la Pathologie, la Chimie et la Cytologie des Foies gras (journées scientifiques du CNERNA, I953). Depuis, de nombreux travaux ont été publiés sur ce sujet. Dans la plupart des cas, ce sont des considérations médicales qui sont à leur origine et le Rat sert de matériel expérimental. Plusieurs revues bibliographiques récentes rassemblent les résultats obtenus (LoMBARDI, I966; CI,ÉMENT, I967).

( $)$ Communication présentée aux Journées d'Étude sur l'élevage et la production de l'Oie. Jouy-en-Josas mai 1967 . 
La production du " foie gras " chez l'oie constitue un autre aspect des stéatoses. En effet, l'engraissement est provoqué par une alimentation forcée : le gavage. Le régime utilisé, la méthode mise en œuvre et la durée du traitement sont fixés par la coutume, car on ignore tout des processus métaboliques qui aboutissent à la formation du foie gras. Pour cerner le problème, nous étudions les modifications qui surviennent au cours du gavage. Ce premier essai, réalisé sur un petit nombre d'animaux, est consacré à l'analyse de divers constituants hépatiques: lipides, protéines et acides nucléiques.

\section{MATÉRIEL ET MÉTHODES}

\section{Animaux et régimes}

Les oies utilisées sont de race Masseube et âgées de 4 mois. Elles sont élevées au Domaine expérimental d'Artiguères, suivant les méthodes ordinairement utilisées dans ce domaine (MONACHON, I966). Quatre semaines avant le gavage, les animaux reçoivent à volonté un régime constitué de $4 \circ \mathrm{p}$. 100 de maĩs, $20 \mathrm{p}$. 100 d'orge, $20 \mathrm{p}$. I00 de farine de viande et $20 \mathrm{p}$. 100 de tourteau d'arachide. Pendant cette période (prégavage) du fourrage vert, renouvelé quotidiennement, est mis à leur disposition. Pendant le gavage, les oies sont nourries avec un mélange cuit à la vapeur et salé de $95 \mathrm{p}$. roo de maís entier et $5 \mathrm{p}$. Ioo de saindoux. Chaque animal reçoit ainsi trois repas par jour de $200 \mathrm{~g}$ environ de produit sec.

Deux oiseaux sont sacrifiés à la fin de la période de prégavage, deux autres après 12 jours de gavage et les deux derniers après $2 \mathrm{I}$ jours. Les foies sont prélevés immédiatement après le sacrifice (section des artères carotides et des veines jugulaires) et congelés à $-5^{\circ} \mathrm{C}$.

\section{Méthodes analytiques} tuelles.

- La matière sèche et les matières azotées totales sont déterminées selon les techniques habi-

- Les lipides sont extraits à froid par le mélange chloroforme-méthanol-eau. Les séparations chromatographiques sont réalisées sur couche mince de silicagel $\mathrm{G}$ pour les graisses neutres et sur Kieselgel R pour les phospholipides (SKIPSKI et $a l$., I963).

- Les acides gras sont analysés en chromatographie gaz-liquide sur DEGS à $176^{\circ} \mathrm{C}$.

- Le phosphore est mesuré par colorimétrie du complexe phosphomolybdovanadique.

- Les acides nucléiques sont extraits selon la méthode de SchMIDT et TANNHAUSER et déterminés à partir de la concentration en bases puriques (DuRAND et al., I965).

\section{RÉSULTATS ET DISCUSSION}

L.es foies obtenus en fin de gavage sont de taille et de qualité moyennes. Cependant l'état de stéatose est suffisant et malgré le faible nombre de répétitions dans chaque lot, il est possible d'étudier l'influence du gavage sur la composition du tissu hépatique.

Nous présentons successivement des résultats d'analyse portant sur la composition globale du foie, sur celle des lipides et des acides nucléiques hépatiques.

\section{Composition globale du foie}

Dans le tableau $I$, nous indiquons à la fois le pourcentage et la quantité totale de chaque constituant contenu dans l'organe : matière sèche, lipides, protéines $(\mathrm{N} \times 6,25)$. On note évidemment une accumulation très importante de lipides. Au 
cours du gavage, la quantité totale est multipliée par un facteur voisin de 20 dans le meilleur cas. Mais l'hétérogénéité des valeurs obtenues est si grande que le second des foies prélevés en fin d'expérience est apparemment moins gras que ceux prélevés au cours du gavage. La matière sèche présente les mêmes variations.

TABLEAU I

Caractéristiques analytiques des foies en fonction de la durée du gavage

\begin{tabular}{|c|c|c|c|c|c|c|c|c|}
\hline \multirow{2}{*}{ Traitement } & \multirow{2}{*}{ Oie } & \multirow{2}{*}{$\begin{array}{l}\text { Poids frais } \\
\text { (g) }\end{array}$} & \multicolumn{2}{|c|}{ Matières sèches } & \multicolumn{2}{|c|}{ Lipides } & \multicolumn{2}{|c|}{$\begin{array}{l}\text { Protéines } \\
(\mathrm{N} \times 6,25)\end{array}$} \\
\hline & & & $\begin{array}{c}\text { Tctal } \\
\text { (g) }\end{array}$ & $\begin{array}{l}\% \text { poids } \\
\text { frais }\end{array}$ & $\begin{array}{c}\text { Total } \\
\text { (g) }\end{array}$ & $\begin{array}{l}\% \text { poids } \\
\text { frais }\end{array}$ & $\begin{array}{c}\text { Total } \\
\text { (g) }\end{array}$ & $\begin{array}{c}\% \text { poids } \\
\text { frais }\end{array}$ \\
\hline \multirow{2}{*}{ Avant gavage .... } & $\mathbf{E}$ & 135 & 39 & 29,10 & 3,0 & $\begin{array}{ll}1 & \\
& 2,25\end{array}$ & 26,5 & 19,61 \\
\hline & $\mathbf{F}$ & 145 & 45 & 30,73 & 7,9 & 5,45 & 30,2 & 20,83 \\
\hline \multirow{2}{*}{ A mi-gavage ..... } & $\mathrm{C}$ & 305 & 155 & 44,40 & 76,8 & 25,2 & 45,2 & 14,85 \\
\hline & $\mathrm{D}$ & 400 & 192 & 47,90 & 134,7 & 37,7 & 62,0 & 15,51 \\
\hline \multirow{2}{*}{ En fin de gavage. } & $\mathbf{A}$ & - 390 & 212 & 54,50 & 150,0 & 38,5 & 39,9 & 10,21 \\
\hline & B & 237 & 107 & 45,20 & 59,0 & 24,8 & 38,4 & 16,20 \\
\hline
\end{tabular}

L'hypertrophie hépatique ne correspond pas à une simple surcharge lipidique. Si la teneur en protéine tend à diminuer au cours du gavage, la quantité totale contenue dans l'organe augmente fortement et cela, malgré un régime relativement défcient en substances azotées; tant sur le plan quantitatif que qualitatif. Cette néosynthèse de protéines a lieu au début du gavage. D'ailleurs, nous constaterons que l'activité anabolique est de règle à cette époque et qu'elle concerne à des degrés variables, tous les constituants cellulaires analysés.

\section{Nature et composition des lipides hépatiques}

La répartition des différentes fractions lipidiques fait également apparaître des différences entre les trois lots (tabl. 2).

La quantité de cholestérol totale s'élève considérablement au cours du gavage. Elle est décuplée à la fin de l'expérience. Cette augmentation porte surtout sur la forme estérifiée, dont la réserve hépatique est multipliée 50 fois environ, tandis que le cholestérol libre est seulement doublé. Il s'ensuit une élévation considérable du taux d'estérification du cholestérol, avec pour conséquence un accroissement très marqué de la valeur du rapport $\frac{\text { cholestérol estérifié }}{\text { cholestérol total }}$.

Cependant, la quantité de cholestérol retenue par le foie reste faible, eu égard à l'importance de la stéatose. 
En définitive, les lipides du foie gras sont une des graisses animales parmi les pluss pauvres en cholestérol.

TABLEAU 2

Composition des lipides hépatiques

\begin{tabular}{|c|c|c|c|c|c|c|c|c|c|c|c|}
\hline & \multicolumn{2}{|c|}{$\begin{array}{l}\text { Phospho- } \\
\text { lipides }\end{array}$} & \multicolumn{2}{|c|}{$\begin{array}{c}\text { Trigly- } \\
\text { cérides }\left(^{\left({ }^{2}\right)}\right.\end{array}$} & \multicolumn{2}{|c|}{$\begin{array}{l}\text { Cholestérol } \\
\text { total }\end{array}$} & \multicolumn{2}{|c|}{$\begin{array}{l}\text { Cholestérol } \\
\text { libre }\end{array}$} & \multicolumn{2}{|c|}{$\begin{array}{l}\text { Cholestérol } \\
\text { estérifié }\end{array}$} & \multirow[t]{2}{*}{$\frac{\text { Chol. estér }}{\text { Chol. total }}$} \\
\hline & $\begin{array}{l}\text { Total } \\
(\mathrm{g})\end{array}$ & $\begin{array}{l}\% \text { des } \\
\text { lipides }\end{array}$ & $\begin{array}{c}\text { Total } \\
\text { (g) }\end{array}$ & $\begin{array}{l}\% \text { des } \\
\text { lipides }\end{array}$ & $\begin{array}{l}\text { Total } \\
(\mathrm{g})\end{array}$ & $\begin{array}{l}\% \text { des } \\
\text { lipides }\end{array}$ & $\begin{array}{c}\text { Total } \\
(\mathrm{g})\end{array}$ & $\begin{array}{l}\% \text { des } \\
\text { lipides }\end{array}$ & $\begin{array}{l}\text { Total } \\
(\mathrm{g})\end{array}$ & $\begin{array}{l}\% \text { des } \\
\text { lipides }\end{array}$ & \\
\hline 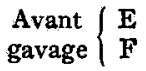 & $\begin{array}{l}2,07 \\
4,60\end{array}$ & $\begin{array}{l}69,0 \\
58,2\end{array}$ & $\begin{array}{l}0,80 \\
3,00\end{array}$ & $\begin{array}{l}26,7 \\
38,0\end{array}$ & $\begin{array}{l}0,21 \\
0,33\end{array}$ & $\begin{array}{l}7,14 \\
4,21\end{array}$ & $\begin{array}{l}0,17 \\
0,27\end{array}$ & $\begin{array}{l}5,90 \\
3,50\end{array}$ & $\begin{array}{l}0,04 \\
0,06\end{array}$ & $\begin{array}{l}\mathbf{1}, 24 \\
0,71\end{array}$ & $\begin{array}{l}0,190 \\
0,180\end{array}$ \\
\hline Mi- $\left\{\begin{array}{l}\mathrm{C} \\
\mathrm{D}\end{array}\right.$ & $\begin{array}{r}8,30 \\
13,00\end{array}$ & $\begin{array}{r}10,8 \\
9,7\end{array}$ & $\begin{array}{r}67,3 \\
119,7\end{array}$ & $\begin{array}{l}87,5 \\
88,9\end{array}$ & $\begin{array}{l}1,17 \\
2,00\end{array}$ & $\begin{array}{l}1,52 \\
1,49\end{array}$ & $\begin{array}{l}0,43 \\
0,56\end{array}$ & $\begin{array}{l}0,56 \\
0,42\end{array}$ & $\begin{array}{l}0,74 \\
1,44\end{array}$ & $\begin{array}{l}0,96 \\
1,07\end{array}$ & $\begin{array}{l}0,630 \\
0,720\end{array}$ \\
\hline 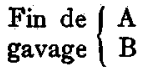 & $\begin{array}{l}6,45 \\
8,95\end{array}$ & $\begin{array}{r}4,3 \\
15,2\end{array}$ & 140,0 & 93,2 & 3,60 & 2,40 & 0,51 & 0,34 & 3,08 & 2,06 & 0,855 \\
\hline
\end{tabular}

(1) Obtenus par différence entre les lipides totaux et la somme des phospholipides et du cholestérol total.

Comme chez la piupart des homéothermes, les phospholipides représentent les 2/3 des lipides à la fin de la période de prégavage : il n'existe alors aucun signe de stéatose. En fin d'expérience, cette proportion s'abaisse à Io p. Ioo.

Le gavage entraîne donc une diminution très marquée du pourcentage des phospholipides dans le foie. Les proportions des différents phospholipides sont peu modifiées par l'engraissement forcé comme le montre le tableau 3. Les lipides de constitution du foie ne semblent donc pas altérés.

TABLEAU 3

Composition des phospholipides hépatiques

\begin{tabular}{c|c|c|c|c|c|c|c}
\hline \hline & $\begin{array}{c}\text { Cardia- } \\
\text { lipides } \\
\text { Acides } \\
\text { phospha- } \\
\text { tidiques }\end{array}$ & $\begin{array}{c}\text { Phospha- } \\
\text { tidyl- } \\
\text { éthanol- } \\
\text { amine }\end{array}$ & $\begin{array}{c}\text { Phospha- } \\
\text { tidyl- } \\
\text { sérine }\end{array}$ & $\begin{array}{c}\text { Phospha- } \\
\text { tidyl- } \\
\text { inosine }\end{array}$ & $\begin{array}{c}\text { Phospha- } \\
\text { tidyl- } \\
\text { choline }\end{array}$ & $\begin{array}{c}\text { Sphingo- } \\
\text { myéline }\end{array}$ & $\begin{array}{c}\text { Lyso- } \\
\text { lécithine }\end{array}$ \\
\hline Avant gavage ........ & 1,0 & 28,2 & 3,2 & 5,9 & 56,6 & 4,6 & 1,0 \\
\hline En fin de gavage ... & 1,3 & 25,1 & 5,5 & 7,4 & 54,0 & 5,1 & 1,2 \\
\hline \hline
\end{tabular}

Toutefois, la quantité totale de ces composés augmente, comme les protéines, au début du gavage.

Les triglycérides constituent donc la majeure partie des graisses accumulées dans le foie. En fin d'expérience ils représentent $90 \mathrm{p}$. Ioo des lipides contre moins de 30 p. roo avant le gavage. 
Dans le tableau 4 sont rassemblées les compositions en acides gras des principaux constituants lipidiques.

Le gavage a peu d'effet sur la composition des phosphatidylcholines (lécithines) et des phosphatidyléthanolamines (céphalines). Il en est tout autrement des triglycérides. Dans ces constituants, l'acide linoléique tend à disparaître. Les trois acides gras les plus abondants en fin d'engraissement sont les acides palmitique, oléique et stéarique : le degré de saturation est très élevé et ne diffère guère de celui d'un suif.

\section{TABLEAU 4}

Composition en acides gras des principaux lipides hépatiques

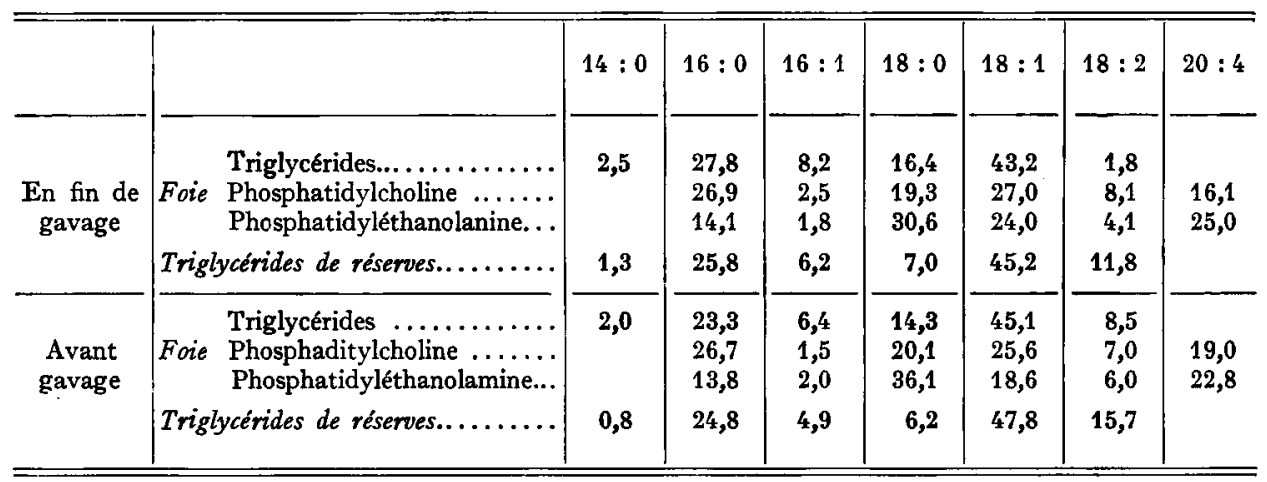

Ce fait exceptionnel chez les oiseaux, oppose l'état de stéatose résultant du gavage à celui qui procède du jeûne ou de la réalimentation chez le poulet (FEIGENBaUm et FisHER, I967).

Ces caractéristiques plaident en faveur d'une synthèse in situ de ces constituants à partir des glucides du régime. Il est peu probable que les acides gras des réserves adipeuses périphériques soient mobilisés vers le foie et participent aux synthèses de triglycérides dans cet organe. En effet, les proportions des acides gras dans les tissus demeurent constantes pendant le gavage et se différencient nettement de celles observées dans le foie (tabl. 4). En outre, on sait depuis longtemps (FLEURET, I953) que ces dépôts augmentent au cours du gavage. C'est dire que pendant cette période, l'activité du tissu adipeux est plutôt orientée vers la lipogenèse que vers la lipolyse.

Par ailleurs, la déficience du régime en protides et facteurs lipotropes doit gêner la formation des lipoprotéines hépatiques et de ce fait le transport des lipides du foie vers la périphérie. La richesse du foie en esters du cholestérol plaide en faveur de cette défaillance du système de transport.

\section{3. Évolution du contenu du foie en acides nucléiques}

Les résultấts d'analyse des acides nucléiques sont rassemblés dans le tableau 5 .

: Le nombre d'animaux étudiés étant trop restreint, nous ne retiendrons, dans cet essai préliminaire, que les modifications les plus marquées.

Entre le commencement et le $12^{\mathrm{e}}$ jour du gavage, on constate une augmentation importante de la quantité totale d'ARN contenue dans l'organe et dans une propor- 
tion moindre de celle de 1 'ADN : les valeurs finales sont inférieures à celles du foie normal pour l'ADN, mais non pour l'ARN.

Le rapport $\frac{A R N}{A D N}$ s'élève donc au début de l'engraissement forcé. Mais du $12^{\mathrm{e}}$ au $2 \mathrm{I}^{\mathrm{e}}$ jour, il demeure invariable. Il en est de même du rapport $\frac{\text { protéines. }}{\text { ADN }}$

Le rapport $\frac{\text { poids frais }}{\text { ADN }}$ croît continuellement : cela s'explique par l'augmentation considérable des dépôts lipidiques.

TABLEAU 5

Evolution du contenu du foie en acides nucléiques

avant et au cours du gavage

\begin{tabular}{|c|c|c|c|c|c|c|}
\hline & \multicolumn{2}{|c|}{ Avant gavage } & \multicolumn{2}{|c|}{ Mi-gavage } & \multicolumn{2}{|c|}{ En fin de gavage } \\
\hline & A & B & c & D & $\mathbf{E}$ & $\mathrm{F}$ \\
\hline 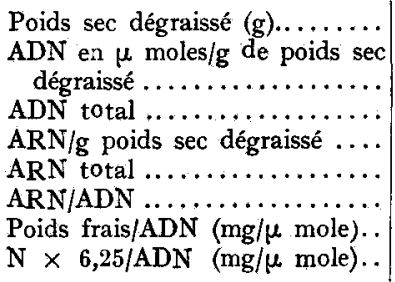 & $\begin{array}{c}35,7 \\
925 \\
91,9 \\
2380 \\
2,57 \\
146 \\
26\end{array}$ & $\begin{array}{c}32,9 \\
994 \\
87,8 \\
2652 \\
2,67 \\
146 \\
28\end{array}$ & $\begin{array}{c}26,4 \\
1148 \\
96,0 \\
4176 \\
3,64 \\
266 \\
35\end{array}$ & $\begin{array}{c}26,3 \\
1339 \\
95,5 \\
4860 \\
3,63 \\
299 \\
35\end{array}$ & $\begin{array}{c}26,1 \\
697 \\
93,5 \\
2496 \\
3,58 \\
459 \\
35\end{array}$ & $\begin{array}{c}22,3 \\
825 \\
94,8 \\
3508 \\
4,25 \\
287 \\
41\end{array}$ \\
\hline
\end{tabular}

Ainsi l'augmentation du contenu total en ADN et en ARN laisse supposer qu'au début du gavage les cellules hépatiques s'accroissent à la fois en nombre et en taille. L'élévation du rapport $\frac{\mathrm{N}}{\mathrm{ADN}}$ suggère que l'accroissement en taille est de loin celui qui prédomine. A la fin du gavage, la quantité d'acides nucléiques contenus dans l'organe n'augmente plus : elle tend même à diminuer en ce qui concerne l'ADN. On peut donc supposer qu'à cette époque, un certain nombre de cellules pourraient être détruites. Pour contrôler cette hypothèse, des études histologiques seraient nécessaires.

$\mathrm{L}_{\text {a valeur élevée du rapport }} \frac{\mathrm{ARN}}{\mathrm{ADN}}$ suggère que l'activité de synthèse du tissu hépatique est exaltée. $L_{a}$ richesse du foie en protéines confirme ce point de vue. Il en est de même des teneurs en phospholipides, cholestérol total et estérifié lorsque ces teneurs sont exprimées, comme le conseille Terroine (I953), en mg par $\mu \mathrm{mol}$ d'ADN (tab1. 6).

En effet, ces teneurs s'élèvent continuellement, la progression étant plus lente pendant la deuxième moitié de la période de gavage.

De ce point de vue, le foie gras de l'oie se singularise des stéatoses d'origine pathologique ou alimentaire observées chez les mammifères (LOMBARDI, Ig66). Les 
lipides ne sont pas accumulés passivement dans l'organe. L'accroissement des synthèses au niveau du tissu hépatique permet d'expliquer l'importance de la stéatose résultant du gavage.

TABLEAU 6

Teneurs du tissu hépatique en certains constituants lipidiques exprimées par rapport au contenu en $A D N$

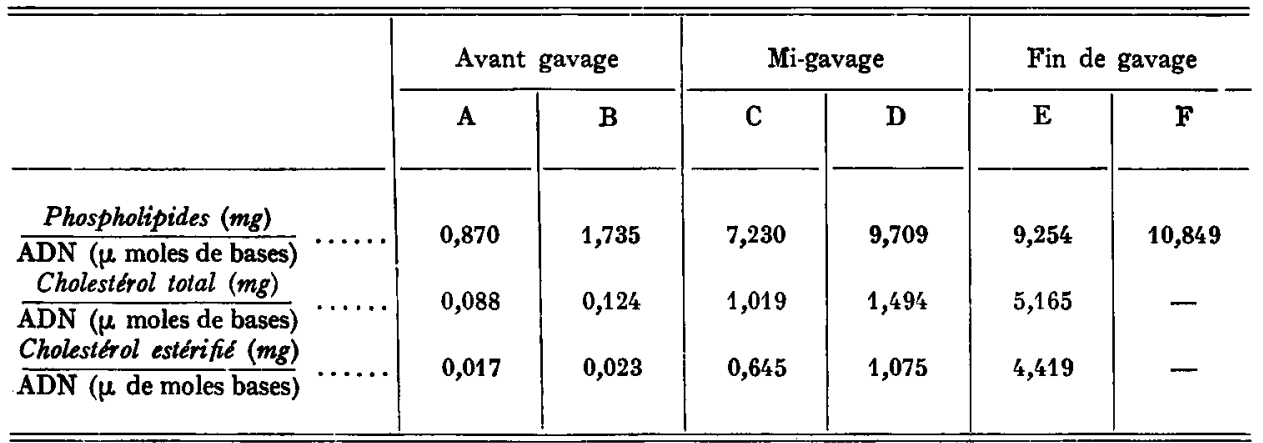

\section{CONCLUSION}

Les modifications qui surviennent dans le tissu hépatique au cours du gavage paraissent relever d'un double mécanisme.

En premier lieu, du fait de l'importance d'un apport alimentaire riche en énergie, 1'animal est conduit à synthétiser une forte quantité de graisse. Cette graisse est constituée essentiellement par des triglycérides qui s'accumulent dans le tissu hépatique. En outre la répartition des acides gras de ces triglycérides est profondément perturbée : c'est l'une des graisses animales les plus pauvres en acide linoléique. Comme les tissus adipeux en demeurent largement pourvus, on peut admettre que les lipides de ces dépôts ne sont pas mobilisés vers le foie. Les triglycérides hépatiques proviennent donc d'une synthèse in situ.

Les autres résultats obtenus dans cet essai révèlent certains aspects de la réaction du tissu hépatique face à l'agression que constitue l'ingestion forcée d'aliment. L'évolution du contenu du foie en acides nucléiques au début du gavage montre que l'organe est le siège à la fois d'une hyperplasie et d'une hypertrophie. Mais cette dernière est prédominante ; elle correspond à une activité cellulaire accrue : synthèse de protéines et de lipides. La prolongation du gavage ne s'accompagne pas d'un accroissement de l'activité du tissu ; il semble au contraire que celle-ci tende quelque peu à diminuer.

Des études complémentaires (activités enzymatiques, examens histologiques, utilisation de radioisotopes) dans le foie et le sérum permettront de préciser ces premiers résultats. 


\section{SUMMARY}

\section{PRELIMINARY NOTE ON CHANGES IN THE BIOCHEMICAL CONSTITUENTS OF LIVER DURING CRAMMING OF GEESE}

Goose livers were analyzed before, during and at the end of cramming. The fat deposit was related to an accumulation of triglycerides. Their fatty acid composition was characterized by an almost complete absence of linoleic acid and a high proportion of stearic acid (table 4). At the same time cholesterol and its esters accumulated in the liver. The proportion of esters was particularly large (table 2). However, the total amount of cholesterol estimated in the liver remained relatively small.

Cramming caused a large increase in the total amount of protein (table r), RNA (table 5) and phospholipids in liver (table 2). The phospholipids were not altered either in their relative proportions or their fatty acid composition (table 4). Fatty acid composition of reserve adipose tissus was totally different from that of the lipids of the liver (table 4); thus adipose tissue does not take part in the formation of the accumulation of fat in the liver of geese. Theese phenomena seem to show that during cramming the size of the cells of the liver increases; they are the site of intense and increasing metabolic activity at least until the middle of the period of cramming. Histological and enzymic studies would be needed to confirm these results.

\section{RÉFÉRENCESS BIBLIOGRAPHIQUES}

Clement J., Clement G., i967. Données récentes de la stéatose hépatique. Cahiers Nutr. Diét., 2, 4I-47.

CoRnatzer W. E., Walser A. H., 1964. Biosynthesis of liver phospholipids during the development of a fatty liver. Proc. Soc. exp. Biol. Med., 116, 893-897.

Durand G., Fauconneau G., PEnot E., 1965. Étude biochimique de la croissance de l'intestin grêle, du foie et de la carcasse du rat; rôles respectifs de la multiplication et du grandissement cellulaires. Ann. Biol. anim. Biochim. Biophys., 5, 165-187.

Feigenbaum A. S., Fisher H., I963. Changes in fatty acid in nutritional fatty degeneration of the liver. I. Effect of starvation. Brit. J. Nutr., 17, $31 \cdot 38$.

Feigenbaum A. S., Fisher H., 1963. Changes in fatty acid in nutritional fatty degeneration of the liver. 2. Effect of realimentation after starvation. Brit. J. Nutr., 17, 39-45.

FleURET P. H., I953. La production du foie gras de volaille destiné à la consommation. Ann. Nutr. Aliment., $7, \mathrm{C}_{97}-\mathrm{C}_{124}$.

Journées scientifiques sur la Physiologie, la Pathologie, la Chimie et la Cytologie des foies gras. I953, Ann. Nutr. Aliment., $7, \mathrm{C} \mathrm{I}-\mathrm{C} 404$.

LoMBardi B., I966. Considerations on the pathogenesis of fatty liver. Lab. Invest., 15, I.

Mayer A., Rathery F., Schaeffer G., Terroine E. F., igr4. Sur les variations expérimentales du chondriome hépatique. Parallélisme entre la composition chimique du tissu et ses aspects cytologiques. C. R. Soc. Biol., 76, 398 .

MoNACHON G., Ig66. Contribution du Domaine expérimental d'Artiguères à l'amélioration de l'élevage de l'oie. XIIIe Congr. Mond. Avic., Kiev, 1966.

Skipski V. P., Peterson R. F., Sanders J., Barclay M., I963. Thin layer chromatography of phospholipids using silica-gel without sulfate binder. J. Lipid Res., 4, 227.

Terroine E. F., Clément G., I953. Composition chimique des foies gras; répartition des lipides et des stérols. Ann. Nutr. aliment., 7 , 280-281. 